\title{
Effect of structural forms on the performance of a jet pump for a deep well jet pump
}

\author{
C. H. Zou, H. Li, P. Tang \& D. H. Xu \\ National Research Center of Pumps and Pumping System \\ Engineering and Technology, Jiangsu University, China
}

\begin{abstract}
A deep well jet pump combines two principles of pumping; that of the centrifugal pump and that of the jet pump. The performance of the jet pump has an impact on the deep well jet pump directly, while previous studies on jet pumps have focused on the structure parameters, which ignored the effect of structural forms such as installation direction and the structural forms of the inlet. In this paper, three turbulence models were used to calculate a 3D single-phase flow field in the jet pump and the results were compared with the experimental data to validate the simulation. Numerical simulations in different gravity fields were carried out to seek the differences of the performance between the horizontal installation and the vertical installation of the jet pump. The optimum throat pipe length formulas were deduced from analysis of the simulation results. Three kinds of structural forms for suction inlet were compared by numerical simulation. The results showed that the efficiency of the vertical inlet was the highest, and some numerical results regarding flow fields and velocity distribution were shown and discussed.

Keywords: deep well jet pump, optimum throat pipe length, gravity field, suction inlet.
\end{abstract}

\section{Introduction}

A deep well jet pump is a kind of high suction device composed of jet pump and power supply through the fittings. At present, experts and scholars have done a lot of research on the internal flow field, the performance of jet pump and comparative design theory of jet pump. The study on jet pump of deep well jet pump is only including performance analysis and calculation. But the effect of structural forms has not been reported yet. The installation direction for a jet pump of deep well jet 
pump can be divided into horizontal and vertical direction. In this paper, by numerical simulation, the performance of the jet pump installed in two different directions and with three kinds of suction inlets was discussed.

\section{Research status}

As the main application for liquid-liquid jet pump, the deep well jet pump is widely used for the river water level regulation, transportation of lubricant, nitroglycerin, jet fuel and etc. Efficient operation of a deep well jet pump depends on the centrifugal pump and jet pump both working in efficiency at the working point.

\subsection{The current research status of jet pump}

A jet pump is a kind of fluid machinery which transfers energy between the working fluid and the suction fluid through turbulent diffusion. The main reason why the efficiency of jet pump is low is the large friction loss from occurring in the process of mixing and the spread of the vortex tube formed in the diffusion loss. In general, the efficiency of jet pump cannot exceed $40 \%$ without regard to the friction loss. In order to make the jet pump working close to high efficient area, the research in the structure and movement performance of jet pump is very necessary.

Through the methods of the theoretical calculation, experiment and numerical simulation, the research on the performance of jet pump is mainly about the key parameters of jet pump including the angle of the nozzle shape and area, contraction angle, throat pipe area and length, diffusion tube length and angle, throat pipe mouth distance of nozzle and throat pipe and reliable design method of the jet pump. The study found that the influence of area ratio has the largest influence. The parameters have an optimum value scope when the jet pump area ratio is fixed .In recent research, the performance of jet pump has improved by the new type of nozzle structure including multiple, impulse nozzles, adjustable nozzles and annular jet pump.

\subsection{The current research status of deep well jet pump}

A deep well jet pump is referred to a device composed by a jet pump and a centrifugal pump as power supply through the connection of pipe which can draw more than $10 \mathrm{~m}$ height. At present, the common machines used in water suction include the jet pump device, the long axis of the submersible electric pump and the deep well pump. While submersible electric pump cost higher and its radial dimension is bigger to the narrow wells. In the Midwest region, submersible electric pump can't be used for lacking of electricity, The long axis deep well pump as rotating machinery, manufacturing requirements and installation accuracy is high, so the cost is higher than others. The deep well jet pump not only meets the requirements of suction height and efficiency, and can also be driven by diesel. 
Relative to the jet pump, the research on performance of deep well jet pump are relatively few at home and abroad. Domestic research is mainly about design and the calculation of the performance. Studies abroad are about the design of deep well jet pump includes the cavitation performance.

\section{Effect of installation direction on performance of jet pump for deep well jet pump}

Commonly, a single jet pump (figure 1) is installed horizontally while the jet pump for a deep well jet pump (figure 2) is vertically installed. In order to compare the performance of the jet pump installed in two directions, different area ratio of the jet pump is simulated and compared.

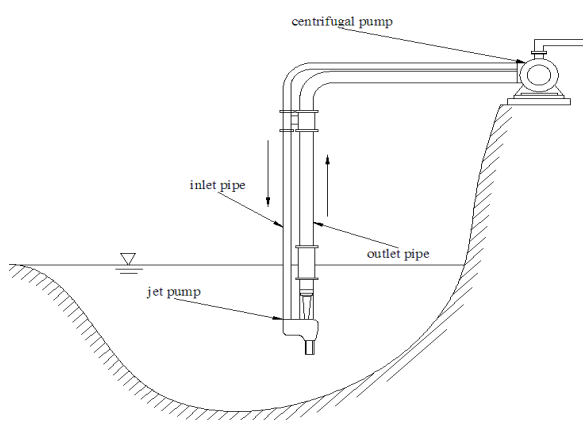

Figure 1: Diagram of the deep well jet pump.

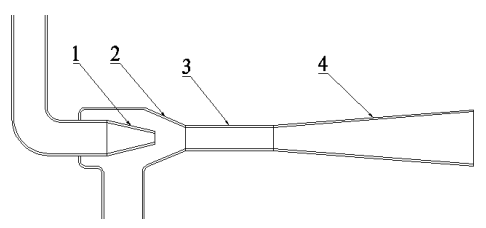

Figure 2: Diagram of the jet pump. 1: nozzle, 2: suction chamber; 3: throat pipe; 4: diffuser.

\subsection{Jet pump with different installation direction of numerical analysis}

The jet pump of XDP505 deep well jet pump was selected as the model pump. The main dimensions are as follows: the area ratio is 4 , the diameter of nozzle outlet is $5 \mathrm{~mm}$, the diameter of throat pipe is $10 \mathrm{~mm}$, the length of throat pipe is $45 \mathrm{~mm}$, the diameter of suction inlet is $30 \mathrm{~mm}$ and the angle of diffuser is $6^{\circ}$. A $3 \mathrm{D}$ model was built by using PRO/E shown in figure 3 .

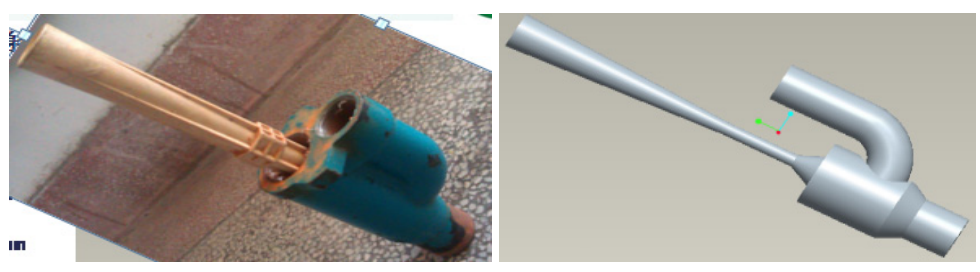

Figure 3: The XDP505 jet pump and the 3D model of it. 
Grids were hexahedral elements and generated by ICEM CFD as the preprocessing software. The grid refinement was made in the place which is near the nozzle and throat pipe where the energy exchange mainly occurring. The grid number was initially made at about 0.15 million and later increased to about 0.21 million to confirm that the results were grid independent. The calculation domain and grid system of the CFD model of the jet pump are shown in figure 4 .
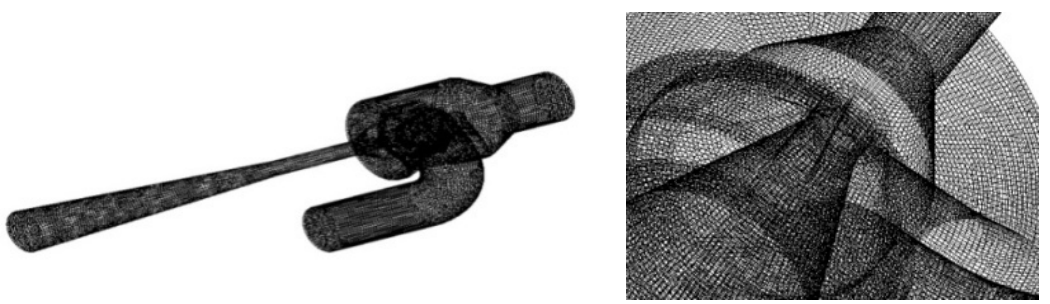

Figure 4: Grids of the jet pump.

The inlet boundary conditions of the two inlets were both set as velocity inlet and the velocity magnitudes were measured in the experiment. The outlet was extended and the boundary condition of which was set as opening added the measured static pressure to allow the flow free development. The wall boundary conditions were set as no slip boundary condition and the surface roughness was set as $0.075 \mathrm{~mm}$. The convergence precision was $1.0 \times 10^{-4}$.

In order to ensure the reliability of the simulation, the performance of XDP505 pump was tested in the laboratory (figures 5 and 6).

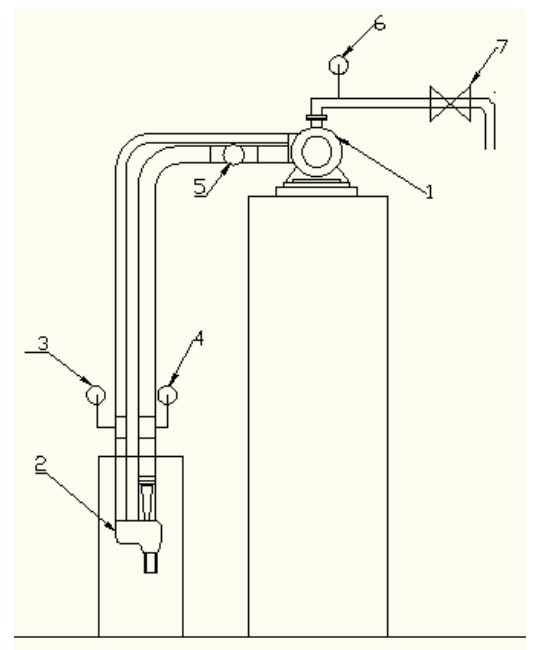

Figure 5: $\quad$ Test-bed of model pump. 1: pump; 2: jet pump; 3, 4, 6: pressure gage; 5: turbine flow meter; 7: valve. 

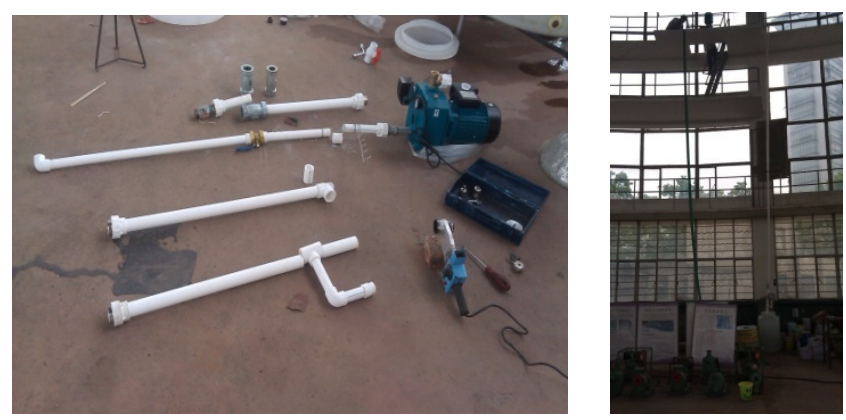

Figure 6: Schematic diagram of model pump test.

Figure 7 shows the $q-h$ curves of test and simulation results by using the three kinds of turbulence model.

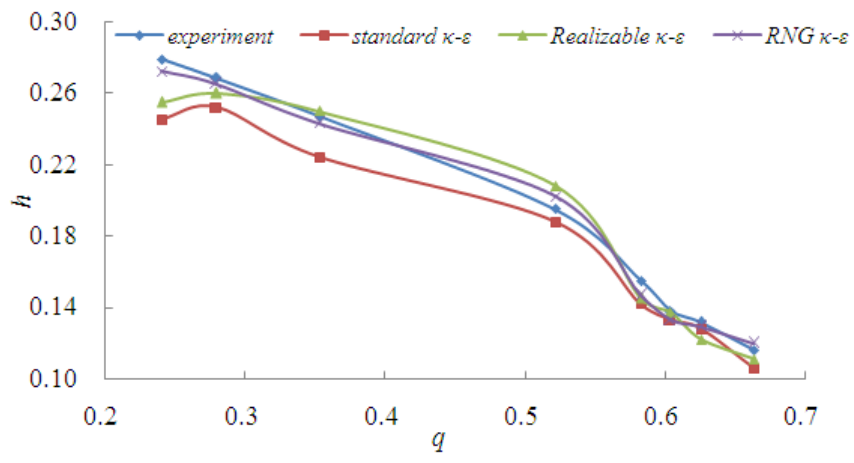

Figure 7: The $\mathrm{q}-\mathrm{h}$ curves of experiment and simulation results.

Table 1 depicts the relative errors between the results of simulation and experiment. It is shown that the result by using RNG $k-\varepsilon$ model is closer to the experiment results than the other methods. It is concluded that the RNG $k$ - $\varepsilon$ model agrees well with the experiment results and it is utilized in the following simulations.

Table 1: The comparison of model jet pump performance with three kinds of turbulence models.

\begin{tabular}{cc}
\hline Turbulence model & Relative error $/ \%$ \\
\hline Standard $k-\varepsilon$ & 6.78 \\
RNG $k-\varepsilon$ & 2.18 \\
Realizable $k-\varepsilon$ & 3.36 \\
\hline
\end{tabular}

Through setting the buoyancy direction, the jet pumps of two installation directions were simulated and the velocity contours were shown in figure 8 . 

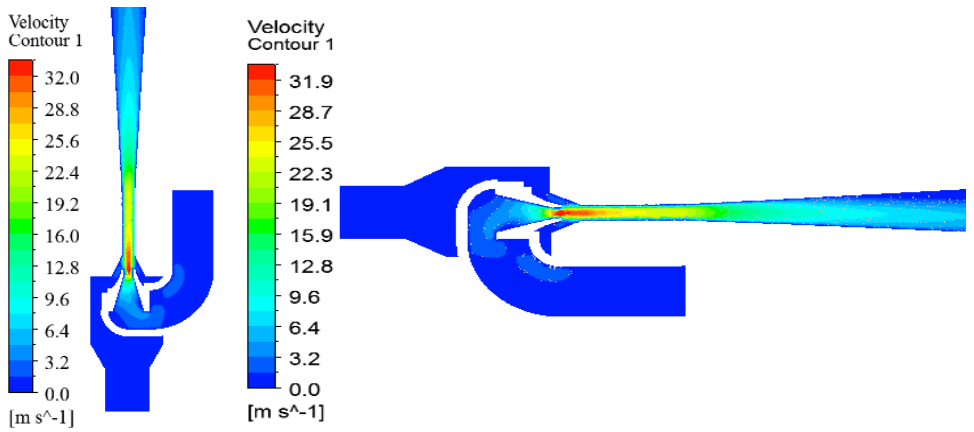

Figure 8: The velocity contours of the two kinds of installation direction.

Figure 9 illustrates that the curves of axial velocity distribution at the two kinds of installation direction. It can be found that the axial velocity of vertical installation is smaller than the one of horizontal installation. This was mainly due to the gravity impact to jet flow field which affects the performance of the jet pump. Therefore, improvement of the design formulas is necessary.

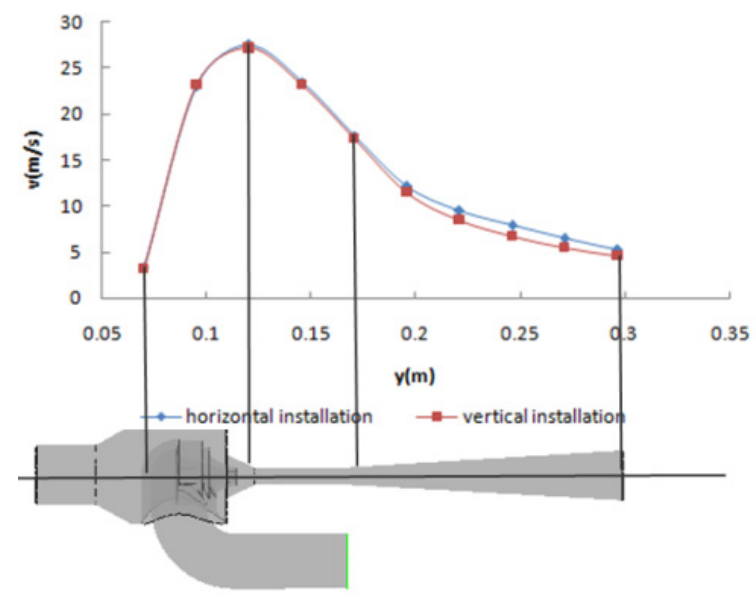

Figure 9: The curves of the axial velocity distribution at the vertical and horizontal installation.

\subsection{Theoretical analysis and fitting the optimum relative throat pipe length formula}

The throat pipe, which is used in mixing the working fluids and the suction fluids, has an important role to the jet pump performance. The too long throat pipe increases the friction losses and the too short throat pipe increases diffusion hydraulic losses. In order to make sure the jet pump work in high efficient area, the reasonable throat pipe length formula deduced for designing the jet pump is 
necessary. On the practical engineering calculation, the length of the throat pipe is usually changed to multiples of throat pipe diameter, using $n$ which is the ratio of throat pipe length and diameter as the relative length of throat pipe. The optimum relative throat pipe length formula (1) was given by Long Xinping [4] through the research through the numerical simulation.

$$
n=0.225 m+5.6
$$

It can be found from the former section that the gravity change has a great influence on flow field in the throat pipe while the optimum length of throat pipe is closely related to the degree of flow nuclear area development in the throat pipe.

The optimum relative throat pipe length of the jet pumps with the highest efficiency installed in two directions in different area ratio was obtained by numerical simulation. XDPM-505 deep well jet pump was chosen as the model pump and different area ratio $m$ was got by changing the nozzle size.

Table 2 shows the data of the optimum throat pipe length in two kinds of direction.

Table 2: The optimum throat pipe length in two kinds of installation direction.

\begin{tabular}{cccccccccc}
\hline$m$ & 3 & 3.5 & 4 & 4.5 & 5 & 5.5 & 6 & 6.5 & 7 \\
\hline$L_{\mathrm{h}}$ & 57.3 & 59.1 & 60.2 & 61.1 & 62.9 & 63.7 & 64.2 & 64.9 & 66.1 \\
$L_{\mathrm{v}}$ & 56.6 & 58.2 & 59.3 & 60.2 & 61.1 & 62.5. & 63.3 & 64.1 & 64.2 \\
\hline
\end{tabular}

The comparison of optimum relative throat pipe length $n$ in two installation direction corresponding different $\mathrm{m}$ is shown in figure 10 .

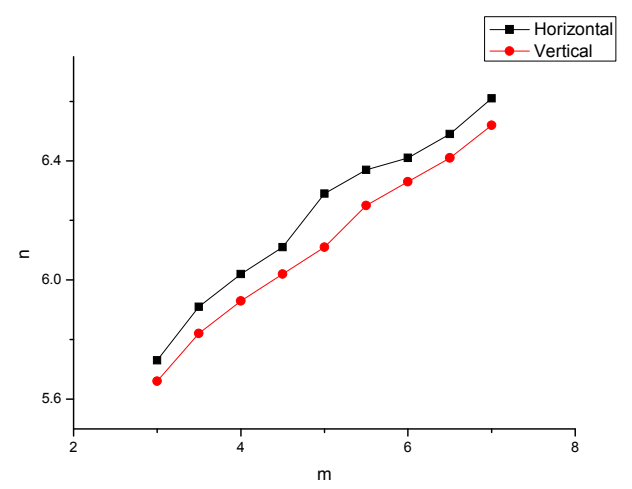

Figure 10: The m-n curves between horizontal and vertical installation.

According to table 2, the formulas of optimum relative throat pipe length was obtained by linear regression, as the following shows:

$$
n_{\mathrm{h}}=0.22 m+5.166
$$




$$
n_{\mathrm{V}}=0.208 m+5.08
$$

Formula (2) is to the horizontal installation and formula (3) is to vertical installation. From the formulas, it is found that formula (2) is similar to the previous formula (1) while formula (3) is distinguished, compared with the formula (1), the intercept of formula (3) is similar to that of formula (1) while the slope of formula (3) is relatively small. The reason is the influence of the gravity field make vertical jet core at the end of the vertical move down.

\section{Impact on performance of the jet pump suction direction}

For the deep well jet pump, the direction of inlet is usually parallel to the direction of the nozzle, and for the study of single jet pump inlet, the direction is vertical or 45 degree angle to the direction of the nozzle. While the conclusion and data of research on single jet pump are used by the study of the deep well jet pump that makes the study of deep well jet pump and the practical situation different. So the study of the jet pump inlet direction is very necessary. It's significant for the precise calculation of the deep well jet pump performance.

\subsection{Numerical simulation of three common inlet direction}

Three kinds of inlets which are vertical, parallel and 45 degree angle to the nozzle were modeled (figure 11). The grids were generated as the former methods and the grid number was about 1.2 million (figure 12). The results of simulation depicted that the inlet which is parallel to the nozzle had better flow field development (figure 13).
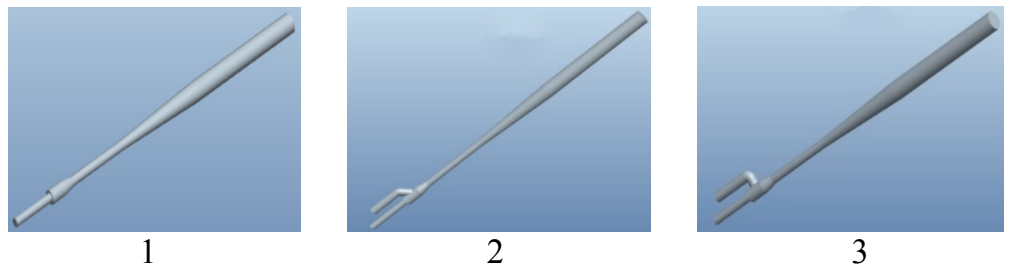

Figure 11: The models of the jet pumps with three different inlets. 1: Parallel to the nozzle; $2: 45^{\circ}$ to the nozzle; 3 : vertical to the nozzle.

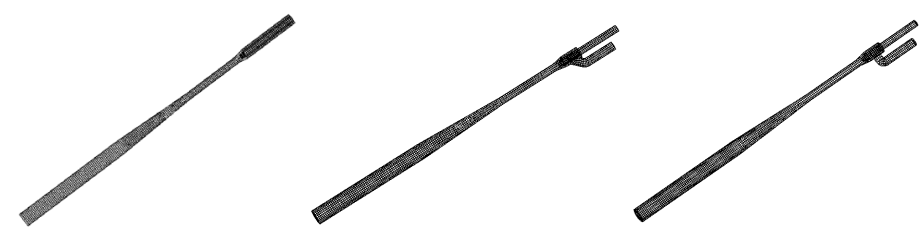

Figure 12: The grids of the jet pumps with three different inlets. 


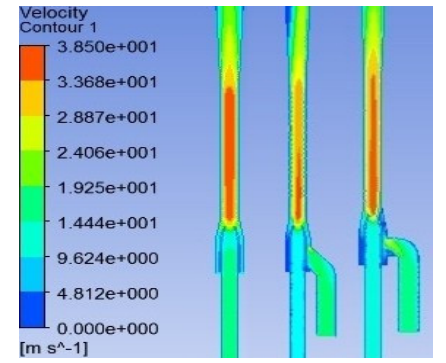

Figure 13: The velocity contour of the jet pumps with three different inlets.

\subsection{The results of comparison and analysis}

Figures 14 and 15 show that the vertical inlet have best performance curve at the same flow rate ratio $q$. It is not only because the direction of inlets of force water and suction water lead to the reduction of the resistance loss, the vertical inlet has best flow field and produces fewest vortexes.

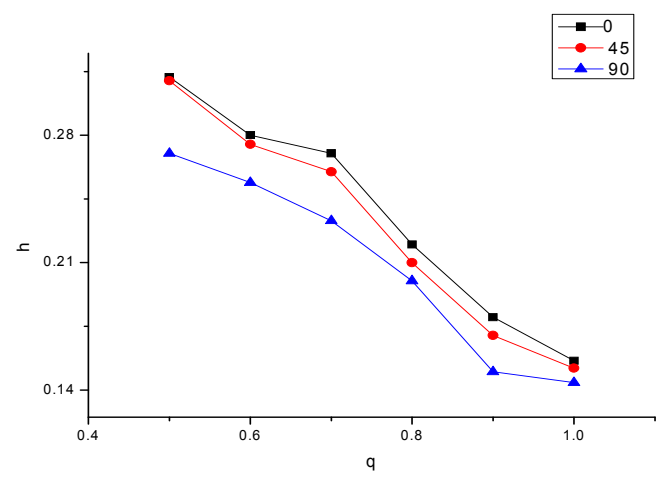

Figure 14: $\mathrm{q}-\mathrm{h}$ curves of three kinds of the jet pumps with three different inlets.

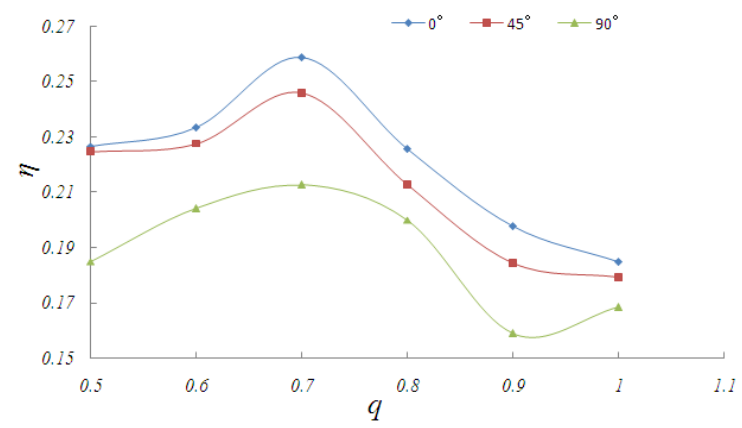

Figure 15: $q-\eta$ curves of three kinds of the jet pumps with three different inlets. 


\section{Conclusion}

In this paper, the performance differences between the single jet pump and the jet pump of the deep well jet pump are analyzed. The jet pumps installed in different direction are simulated and compared.The formula of optimum relative throat pipe length was deduced. The jet pumps with different inlet direction were simulated and the most efficient one was obtained as the vertical inlet.

In order to further study the influence of gravity and improve the overall performance of the deep well jet pump, the whole flow field in numerical simulation of deep well jet pump is needed.

\section{Nomenclature}

$h$ the import and export pressure ratio of jet pump

$m$ the ratio of throat pipe area and the nozzle area

$q$ the ratio of sucked and export flow

$L_{\mathrm{h}} \quad$ the optimum horizontal throat pipe length [m]

$L_{\mathrm{v}}$ the optimum vertical throat pipe length [m]

$n_{\mathrm{h}}$ the optimum relative horizontal throat length

$n_{\mathrm{v}}$ the optimum relative horizontal throat length

\section{References}

[1] Sanger, N.L, An Experimental Investigation of several Low-Area-Ratio Water Jet Pump, Trans. ASME, Journal of the Basic Engineering, Vol. 92, March (1970), 11-20.

[2] Moody, L.F, Friction Factors for Pipe Flow, Trans. ASME, Vol. 66, Nov (1944), 671-684.

[3] Yukitaka Yamazaki. Effect of Surface Roughness on Jet Pump Performance, JSME International Journal, series B, Vol. 19, No. 4, 2006, 928-932.

[4] Long Xinping. Numerical calculation of optimal length of throat pipe in jet pump. Shuili Xuebao/Journal of Hydraulic Engineering, 10, 2003, 14-18.

[5] Eames I.W. A new prescription for the design of supersonic jet-pumps: the constant rate of momentum change method. Appl. Therm. Eng. 2002, 22, 121-31.

[6] Hemidi A., Henry F., Leclaire S., Seynhaeve J-M., Bartosiewicz Y. CFD analysis of a supersonic air ejector: part 1: experimental validation of singlephase and two-phase operation. Appl. Therm. Eng. 2009, 29, 1523-31.

[7] He S., Li Y., Wang R.Z. Progress of mathematical modeling of ejectors. Renew. Sustain. Energy Rev. 2009, 13, 1760-80.

[8] N.H.G. Mueller, Water jet pump, Journal of the Hydraulics Division, ASCE, 90(3), (1964) 83-113. 